\title{
Perfil Bioquímico e Nutricional de Vegetarianos e Onívoros de um Município de Santa Catarina, Brasil
}

\author{
Louyse Sulzbach Damázio ${ }^{1}$, Raquel Genuino Teixeira Grassi' ${ }^{2}$, Tuany Cardoso Mattos ${ }^{3}$, \\ Danielle Celso ${ }^{4}$, Emanuel de Souza ${ }^{5}$, Eduarda Schultze ${ }^{6}$, Ângela Martinha Bongiolo ${ }^{7}$
}

\begin{abstract}
RESUMO
O objetivo deste estudo foi avaliar marcadores bioquímicos e nutricionais e a associação com a composição corporal de vegetarianos e onívoros do município de Criciúma - Santa Catarina. Foram avaliados: parâmetros plasmáticos, níveis séricos de vitamina B12 (nos vegetarianos), índice de massa corporal, circunferência da cintura, composição corporal, motivos para a dieta vegetariana e tempo de vegetarianismo e questionário socioeconômico. Participaram da amostra 34 indivíduos adultos, sendo 11 ovolactovegetarianos, 11 veganos e 12 onívoros, com idade média de 25,24 anos e predominância do gênero feminino. Na classificação do estado nutricional, $97 \%$ dos avaliados apresentou IMC eutrófico. A circunferência da cintura esteve adequada nos ovolactovegetarianos $(90,9 \%)$, veganos $(81,8 \%)$ e com risco moderado nos onívoros $(33,3 \%)$. Verificou-se que entre os ovolactovegetarianos $(27,3 \%)$ e veganos $(18,2 \%)$ houve excesso de gordura corporal e percentil de massa magra muito baixo $(36,4 \%)$. O colesterol total estava normal em $100 \%$ dos ovolactovegetarianos, $81,8 \%$ dos veganos e nos onívoros apresentou-se alto, em $58,3 \%$. Na glicemia, $50 \%$ dos onívoros estavam com risco aumentado para diabetes, enquanto $100 \%$ dos ovolactovegetarianos e $81,8 \%$ dos veganos apresentaram normoglicemia. Ocorreu maior predominância de indivíduos onívoros $(41,66 \%)$ com hemoglobina abaixo do ideal e indicativo de anemia, contra $27,3 \%$ nos vegetarianos. Já os níveis séricos de vitamina B12 nos vegetarianos estavam dentro dos valores ideais, com uma média em $362,86 \mathrm{pg} / \mathrm{ml}$. Mediante este trabalho podemos concluir que os vegetarianos apresentam diferenças sobre o perfil lipídico, glicêmico e na circunferência de cintura quando comparados aos onívoros.
\end{abstract}

Palavras-chave: Antropometria. Vitamina B12. Colesterol.

BIOCHEMICAL AND NUTRITIONAL PROFILE OF VEGETARIANS AND OMNIVORES IN A MUNICIPALITY IN SANTA CATARINA, BRAZIL

\section{ABSTRACT}

To evaluate biochemical and nutritional characteristics and an association with a body composition of vegetarians and omnivores of the municipality of Criciúma - Santa Catarina. Plasma parameters, serum vitamin B12 levels (in vegetarians), body mass index, waist circumference, body composition, reasons for a vegetarian diet and time of vegetarianism and socioeconomic evaluation were evaluated. Thirty-four adult subjects participated in the study, of which 11 were oactoactovegetarians, 11 were vegans and 12 years old, with a daily average of 25.24 years and female predominance. In the classification of nutritional status, $97 \%$ of the evaluated patients presented a BMI. Surgery ended in $72 \%$ of ovolactovegetarians $(90.9 \%)$, vegans $(81.8 \%)$ and moderate risk $(33.3 \%)$. There was much more than body fat and percentage of lean mass $(36.4 \%)$ among ovolactovegetarians $(27.3 \%)$ and vegans $(18.2 \%)$. The total cholesterol was normal in $100 \%$ of the oactoactovegetarians, $81.8 \%$ of the vegans and in the fruits they were elevated in $58.3 \%$. At glycemia, $50 \%$ of the eggs were rich in diabetes, while $100 \%$ of ovaractovegetarians and $81.8 \%$ vegans met normoglycemia. There is a greater predominance of omnivorous individuals $(41.66 \%)$ with hemoglobin below ideal and indicative of anemia, compared to $27.3 \%$ in vegetarians. Serum levels of vitamin B12 in vegetarians are within the ideal range, with a mean of $362.86 \mathrm{pg} / \mathrm{ml}$. We conclude that vegetarians have differences in lipid plasmatic levels, glycemic and waist circumference when compared to omnivores. Keywords: Anthropometry. Vitamin B12. Cholesterol.

RECEBIDO EM: 17/12/2018

MODIFICAÇÕES SOLICITADAS EM: 13/2/2020

ACEITO EM: 24/4/2020

\footnotetext{
Nutricionista e Doutoranda em Ciências da Saúde, Unidade Acadêmica da Saúde, Universidade do Extremo Sul Catarinense - Unesc, Criciúma, SC. http:// lattes.cnpq.br/2476356014456993. https://orcid.org/0000-0002-0710-2320. louyse3@hotmail.com

Acadêmica do Curso de Nutrição, Unidade Acadêmica da Saúde, Universidade do Extremo Sul Catarinense - Unesc, Criciúma, SC. http://lattes.cnpq. br/9352832095343587.raquelgenuino@gmail.com

Acadêmica do Curso de Nutrição, Unidade Acadêmica da Saúde, Universidade do Extremo Sul Catarinense - Unesc, Criciúma, SC. http://lattes.cnpq. br/5285467750438332. https://orcid.org/0000-0001-9040-7565. tuanycmt@gmail.com

${ }^{4}$ Acadêmica do Curso de Biomedicina, Unidade Acadêmica da Saúde, Universidade do Extremo Sul Catarinense - Unesc, Criciúma, SC. http://lattes.cnpq. br/6063970444158555. https://orcid.org/0000-0003-3994-639X. danielle_celso@hotmail.com

${ }^{5}$ Professor do Departamento de Nutrição, Unidade Acadêmica da Saúde, Universidade do Extremo Sul Catarinense - Unesc, Criciúma, SC. http://lattes.cnpq. br/9116673505022391. https://orcid.org/0000-0001-5806-5243. emanuel@unesc.net

${ }^{6}$ Professora do Departamento de Biomedicina, Unidade Acadêmica da Saúde, Universidade do Extremo Sul Catarinense - UNESC, Criciúma, SC. http://lattes. cnpq.br/6578794984578379. https://orcid.org/0000-0002-2347-9697. dudashultze@gmail.com

Professora do Departamento de Nutrição, Unidade Acadêmica da Saúde, Universidade do Extremo Sul Catarinense - Unesc, Criciúma, SC. http://lattes.cnpq. br/2703645841157687.https://orcid.org/0000-0002-5675-7876.nutangela@hotmail.com
} 


\section{INTRODUÇÃO}

O indivíduo vegetariano é aquele que não consome nenhum alimento cárneo (RIBEIRO et al., 2015). A dieta vegetariana apresenta algumas classificações quanto à inclusão ou não de ovos e/ou laticínios. As definições são: ovolactovegetariano, consome ovos e laticínios e exclui carne e peixes; lactovegetariano, quando consome laticínios e exclui carne, peixes e ovos; ovovegetariano, aquela que consome ovos e exclui carne, peixes e laticínios; e vegetariano estrita, quando não consome nenhum alimento ou produto de origem animal (MELINA et al., 2016).

A dieta vegetariana tem sido associada a uma maior longevidade, por proporcionar menores riscos de desenvolver doenças crônicas como obesidade, doenças cardiovasculares, hipertensão (HAS), diabetes mellitus tipo 2 (DM2) e alguns tipos de cânceres. Alguns estudos demonstram que os benefícios são resultado da redução do consumo de gordura saturada e colesterol, bem como da maior ingestão de vegetais, legumes, grãos e frutas (MARSH et al., 2012). Além disso, os vegetarianos apresentam um índice de massa corporal menor quando comparados aos indivíduos onívoros, ou seja, que consomem todos os grupos alimentares (DE BIESE et al., 2007).

$O$ vegetarianismo tem expandido-se e conquistado maiores adesões e interesse popular. No Brasil, $14 \%$ da população brasileira declarou-se vegetariana, o que representa quase 30 milhões de brasileiros; nas regiões de São Paulo, Curitiba, Recife e Rio de Janeiro este percentual subiu para 16\% (IBOPE, 2018).

Um estudo realizado com adultos onívoros e vegetarianos analisou o metabolismo dos quilomícrons e aspectos qualitativos da lipoproteína de alta densidade (HDL). Foi observado que tanto as dietas ovolactovegetariana e vegana aumentam a retirada plasmática das lipoproteínas aterogênicas. Também foi visto que a dieta vegana aparenta ser mais benéfica na prevenção de aterosclerose, uma vez que, na pesquisa, verificou-se que ela impulsiona a queda dos níveis de lipoproteína de baixa densidade (LDL). Já em relação à $\mathrm{HDL}$, não encontraram diferenças de tamanho tampouco em aspectos qualitativos nos grupos avaliados (VINAGRE, 2010). Corroboram, no mesmo sentido, outros estudos, que informam que uma alimentação vegetariana resulta em valores mais baixos no colesterol total, LDL e triglicerídeos (MELINA et al., 2016). A literatura também mostra que vegetarianos apresentam contagens mais elevadas de glóbulos vermelhos quando comparado aos onívoros, e estes, apresentam níveis mais baixos de hematócrito do que os vegetarianos. Já os níveis de hemoglobina são normais nos dois grupos (SAMBOL et al., 2009 ).

Torna-se extremamente importante avaliar os efeitos da dieta vegetariana em diferentes regiões e faixas etárias. Ao que tudo indica, a alimentação à base de alimentos vegetais pode influenciar na composição corporal e em exames bioquímicos. Desse modo, este trabalho é relevante por abordar um tema que está em crescimento no meio científico e médico. O estudo foi desenvolvido a fim de elucidar e comparar as influências da dieta vegetariana (ovolactovegetariana e vegana) e onívora sobre os parâmetros de composição corporal e parâmetros bioquímicos de indivíduos da cidade de Criciúma, Santa Catarina, Brasil.

\section{MATERIAIS E MÉTODOS}

Este é um estudo descritivo, quantitativo, de temporalidade transversal, com amostragem por conveniência. Para atender os objetivos da pesquisa, a população do presente estudo consistiu-se em dois grupos: indivíduos vegetarianos atendidos em um consultório de nutrição do município de Criciúma; e onívoros convidados a participar da pesquisa que apresentavam perfil sociográfico semelhante aos vegetarianos.

O convite para a pesquisa foi realizado por e-mail e a coleta de dados feita no período de março a abril de 2018 nas dependências das Clínicas Integradas da Universidade do Extremo Sul Catarinense (Unesc). A amostra constituiu de 34 indivíduos, sendo 22 vegetarianos e 12 onívoros. Foram incluídos neste estudo vegetarianos (com dieta regular há pelo menos seis meses) e onívoros atendidos até o ano de 2017, de ambos os sexos, com idade entre 18 e 60 anos, passíveis de serem avaliados antropometricamente e de responderem aos questionários.

A primeira etapa da entrevista foi composta pela avaliação da condição socioeconômica, quando foi adotado o questionário Critério de Classificação Econômica Brasil da Associação Brasileira de Empresa de Pesquisa (ABEP, 2008), classificando em seis categorias socioeconômicas, sendo as classes A, B1, B2, C1, C2 e D-E. Posteriormente ao preenchimento dos questionários sobre alimentação vegetariana, foi feito a avaliação antropométrica de peso, altura, circunferência abdominal e aferição das medidas das dobras cutâneas. Em seguida, procedeu-se a coleta de sangue por um profissional biomédico por intermédio de punção venosa. Também foi realizada a análise de 
prontuários para coleta de dados dos níveis de vitamina B12 (cobalamina) dos pacientes com dieta vegetariana.

Os dados de peso foram obtidos por meio da balança da marca Tanita ${ }^{\circledR}$, Tanita Corporation, Tóquio, Japão - modelo Ironman com capacidade de 150 quilos com leitor de bioimpedância acoplado. Para a aferição do peso, a balança foi colocada em uma superfície plana; o indivíduo ficou em posição reta, em pé, no centro da balança, descalço e sem roupas e acessórios pesados que pudessem intervir nos resultados. A estatura foi obtida por intermédio do estadiômetro marca Sanny ${ }^{\circledR}$, American Medical Brasil, SBC, SP - em escala milimétrica $2.000 \mathrm{~mm}$. Para aferição da estatura, o indivíduo posicionou-se em pé, com a cabeça e olhar em linha reta, braços estendidos ao lado do corpo com as palmas das mãos voltadas para a coxa, ombros relaxados, calcanhares e joelhos juntos, pernas retas e pés descalços. A mensuração foi finalizada na obtenção do ponto máximo após ocorrer a inspiração com a postura ereta (MARTINS, 2008).

A análise da composição corporal foi feita mediante o protocolo de Pollock e Wilmore (1993). Realizaram-se medidas das dobras cutâneas $(\mathrm{mm})$ com o auxílio de um adipômetro (Lange, EUA) com leitura de $0,5 \mathrm{~mm}$. Os pontos de medida foram: peito, tríceps, subescapular, subaxilar, suprailíaca, abdome e coxa. Após a realização das medidas, foi efetuado o cálculo para verificar a porcentagem de gordura corpórea segundo Pollock e Wilmore (1993).

A medida da circunferência da cintura foi aferida com uma fita métrica (precisão de 0,1 cm). O participante ficou em pé, com a remoção parcial da roupa superior até abaixo do tórax, os músculos abdominais relaxados, os braços ao lado do corpo e os pés juntos. A circunferência foi coletada a partir do ponto médio da cintura, que está localizado entre a última costela e a suprailíaca. Em seguida, posicionou-se a fita métrica confortavelmente no plano horizontal ao redor do abdome, na altura do ponto médio. A leitura foi feita após a expiração normal do avaliado. A classificação foi realizada de acordo com os valores de referência da Organização Mundial da Saúde em 1998 (OMS) (WHO, 1998).

Todos os exames bioquímicos foram realizados em parceria com o Laboratório de Ensino em Análises Clínicas (Lenac) do curso de Biomedicina da Universidade do Extremo Sul Catarinense (Unesc). Foram coIhidos os exames bioquímicos de hemograma e perfil lipídico. Para o dia da pesquisa os voluntários foram orientados a permanecer em jejum, de no mínimo, 9 horas. A coleta foi realizada em dois tubos, um para análise do perfil lipídico e glicêmico e outro tudo a vácuo com anticoagulante ácido etilenodiaminotetracético (EDTA K2) para análise hematológica. Em seguida, as amostras foram levadas para o laboratório, homogeneizadas e centrifugadas por 10 minutos a velocidade de $3.600 \mathrm{rpm}$ em temperatura ambiente.

Os dados foram tabulados e analisados com o auxílio do programa IBM SPSS Statistics versão 22.0 e apresentados como frequência absoluta e relativa, média e desvio padrão da média ou mediana e intervalo interquartil, a depender da normalidade da distribuição dos dados.

A pesquisa foi submetida ao Comitê de Ética em Pesquisa da Unesc, respeitando a Resolução no 466 de 2012 do Conselho Nacional de Saúde (CNS). A coleta dos dados somente foi iniciada após a aprovação do projeto pelo Comitê de Ética mediante parecer número 2.399.924.

\section{RESULTADOS}

Dos 22 vegetarianos, 11 apresentaram dieta ovolactovegetariana e 11 vegana. A média da idade dos pesquisados foi de $25,24 \pm 4,75$ anos e prevalência do gênero feminino. Em relação à escolaridade, a maioria possuía Ensino Superior Incompleto. Predominou-se entre os grupos as classes B1 e B2 da amostra, enquanto a classe $A$ prevaleceu no grupo dos veganos (Tabela 1). No que se refere à motivação em adotar a dieta vegetariana/vegana, a maior parte relatou ser motivada pela ética. No que diz respeito ao tempo de vegetarianismo, prevaleceu entre 6 a 12 meses (Tabela 2).

Observou-se predominância da média do índice de massa corporal (IMC) e da circunferência da cintura (CC) dentro do adequado em ambos os grupos presentes no estudo (Tabela 3).

$\mathrm{Na}$ classificação do estado nutricional dos grupos, observou-se, na maior parte dos pesquisados, IMC eutrófico, tendo apenas no grupo dos onívoros $8,3 \%$ de sobrepeso. A CC adequada predominou mais entre os ovolactovegetarianos e veganos (Tabela 4).

Quanto à composição corporal, a maioria apresentou percentual adequado, com resultados semeIhantes entre os grupos, porém verificou-se que os ovolactovegetarianos e veganos mostraram excesso de gordura corporal. No que se refere à composição de massa magra, a maior parte dos participantes apresentou CMB normal, havendo pouca diferença nos três grupos. Já em relação à $A M B$, que reflete a massa muscular livre de gordura e osso, 53\% da amostra avaliada está dentro do adequado, no entanto foi ob- 
Tabela 1 - Características socioeconômicas de indivíduos onívoros, ovolactovegetarianos e veganos. Criciúma - Santa Catarina. 2018

\begin{tabular}{|c|c|c|c|c|}
\hline Variável & $\begin{array}{c}\text { Onívoros } \\
\begin{array}{c}(n=12) \\
f(\%)\end{array}\end{array}$ & $\begin{array}{c}\text { Ovolacto } \\
\text { (n=11) } \\
f(\%)\end{array}$ & $\begin{array}{c}\text { Veganos } \\
\begin{array}{c}(n=11) \\
f(\%)\end{array}\end{array}$ & $\begin{array}{l}\begin{array}{l}\text { Total } \\
(n=34)\end{array} \\
f(\%)\end{array}$ \\
\hline \multicolumn{5}{|l|}{ Gênero } \\
\hline Feminino & $11(91,7)$ & $9(81,8)$ & $10(91,0)$ & $30(88,2)$ \\
\hline Masculino & $1(8,3)$ & $2(18,2)$ & $1(9,0)$ & $4(11,8)$ \\
\hline \multicolumn{5}{|l|}{ Escolaridade } \\
\hline Superior incompleto & $9(75,0)$ & $5(45,4)$ & $4(36,4)$ & $18(52,9)$ \\
\hline Superior completo & $1(8,3)$ & $2(18,2)$ & $4(36,4)$ & $7(20,6)$ \\
\hline \multicolumn{5}{|l|}{ Pós-Graduação incompleta } \\
\hline Pós-Graduação completa & $1(8,3)$ & $3(27,3)$ & $1(9,0)$ & $5(14,7)$ \\
\hline \multicolumn{5}{|l|}{ Classificação Abep } \\
\hline Classe A & $1(8,3)$ & $1(8,3)$ & $2(18,2)$ & $4(11,8)$ \\
\hline \multicolumn{5}{|l|}{ Classe B1 } \\
\hline Classe B2 & $1(8,3)$ & - & $3(27,3)$ & $4(11,8)$ \\
\hline \multirow[t]{3}{*}{ Classe C1 } & $5(41,7)$ & $5(45,4)$ & $3(27,3)$ & $13(38,2)$ \\
\hline & $6(50,0)$ & $5(45,4)$ & $5(45,4)$ & $16(47,0)$ \\
\hline & - & $1(8,3)$ & - & $1(3,0)$ \\
\hline
\end{tabular}

Fonte: Elaborada pelos autores.

Tabela 2 - Motivos de adoção da dieta vegetariana e tempo de vegetarianismo entre indivíduos ovolactovegetarianos e veganos. Criciúma - Santa Catarina. 2018

\begin{tabular}{|l|c|}
\hline \multicolumn{1}{|c|}{ Variável } & $(\mathbf{n = 2 2 )}$ \\
& $\boldsymbol{f}(\mathbf{\%})$ \\
\hline Motivação & $13(59,1)$ \\
Ética & $4(18,2)$ \\
Meio ambiente & $1(4,5)$ \\
Não gosta de carne & $1(4,5)$ \\
Religião/espiritualidade & - \\
Saúde & - \\
Família & - \\
loga & - \\
Filosofia & $3(13,6)$ \\
Todos os motivos juntos & \\
Tempo de vegetarianismo & $9(41,0)$ \\
6 a 12 meses & $5(22,7)$ \\
13 a 24 meses & $3(13,6)$ \\
25 a 36 meses & $1(4,5)$ \\
37 a 48 meses & $1(4,5)$ \\
49 a 60 meses & $1(4,5)$ \\
73 a 84 meses & $1(4,5)$ \\
95 a 96 meses & $1(4,5)$ \\
\hline
\end{tabular}

Fonte: Elaborada pelos autores.

Tabela 3 - Dados antropométricos de indivíduos onívoros, ovolactovegetarianos e veganos.

Criciúma - Santa Catarina. 2018

\begin{tabular}{lcccc}
\hline Características* & $\begin{array}{c}\text { Onívoros } \\
(\mathbf{n = 1 2})\end{array}$ & $\begin{array}{c}\text { Ovolacto } \\
(\mathbf{n = 1 1})\end{array}$ & $\begin{array}{c}\text { Veganos } \\
(\mathbf{n = 1 1})\end{array}$ & $\begin{array}{c}\text { Total } \\
(\mathbf{n}=\mathbf{3 4})\end{array}$ \\
\hline Peso $(\mathrm{kg})^{1}$ & $59 \pm 7,96$ & $56,82 \pm 4,86$ & $56,18 \pm 4,20$ & $57,38 \pm 6,10$ \\
Estatura $(\mathrm{m})^{1}$ & $1,65 \pm 0,06$ & $1,66 \pm 0,08$ & $1,61 \pm 0,04$ & $1,64 \pm 0,07$ \\
IMC $\left(\mathrm{kg} / \mathrm{m}^{2}\right)^{1}$ & $21,71 \pm 2,15$ & $20,64 \pm 1,70$ & $21,74 \pm 1,92$ & $21,40 \pm 2,00$ \\
CC $(\mathrm{cm})^{2}$ & $77,67 \pm 7,12$ & $72,64 \pm 7,05$ & $72,82 \pm 6,51$ & $74,47 \pm 7,30$ \\
\hline
\end{tabular}

Fonte: Elaborada pelos autores.

${ }^{*}$ Dados expressos como média \pm desvio padrão (DP). ${ }^{1}$ WHO (1998). ${ }^{2}$ OMS (1995) . 
Tabela 4 - Classificação do estado nutricional de indivíduos onívoros, ovolactovegetarianos e veganos. Criciúma - Santa Catarina. 2018

\begin{tabular}{|c|c|c|c|c|}
\hline Variável & $\begin{array}{c}\text { Onívoros } \\
(n=12) \\
f(\%)\end{array}$ & $\begin{array}{c}\begin{array}{c}\text { Ovolacto } \\
(n=11) \\
f(\%)\end{array} \\
\end{array}$ & $\begin{array}{c}\text { Veganos } \\
(n=11) \\
f(\%)\end{array}$ & $\begin{array}{c}\text { Total } \\
(n=34) \\
f(\%)\end{array}$ \\
\hline \multicolumn{5}{|l|}{$\mathrm{IMC}\left(\mathrm{kg} / \mathrm{m}^{2}\right)^{1}$} \\
\hline Magreza & - & - & - & - \\
\hline Eutrofia & $11(91,7)$ & $11(100)$ & $11(100)$ & $33(97,0)$ \\
\hline Sobrepeso & $1(8,3)$ & - & - & $1(3,0)$ \\
\hline Obesidade Grau I & - & - & - & - \\
\hline Obesidade Grau II & - & - & - & - \\
\hline Obesidade Grau III & - & - & - & - \\
\hline \multicolumn{5}{|l|}{$\mathrm{CC}\left(\mathrm{cm}^{2}\right)^{2}$} \\
\hline Normal & $8(66,7)$ & $10(90,9)$ & $9(81,8)$ & $27(79,4)$ \\
\hline Risco Moderado & $4(33,3)$ & $1(9,1)$ & $2(18,2)$ & $7(20,6)$ \\
\hline Alto Risco & - & - & - & - \\
\hline
\end{tabular}

Fonte: Elaborada pelos autores.

IMC: Índice de Massa Corporal. CC: Circunferência da Cintura.

${ }^{1}$ WHO (1995).

${ }^{2}$ WHO (1998).

Tabela 5 - Classificação da composição corporal de indivíduos onívoros, ovolactovegetarianos e veganos. Criciúma - Santa Catarina. 2018

\begin{tabular}{|c|c|c|c|c|}
\hline Variável & $\begin{array}{c}\text { Onívoros } \\
\begin{array}{c}(n=12) \\
f(\%)\end{array}\end{array}$ & $\begin{array}{c}\text { Ovolacto } \\
\begin{array}{c}(n=11) \\
f(\%)\end{array}\end{array}$ & $\begin{array}{c}\text { Veganos } \\
(n=11) \\
f(\%)\end{array}$ & $\begin{array}{c}\text { Total } \\
(n=34) \\
f(\%)\end{array}$ \\
\hline \multicolumn{5}{|l|}{ \% Gordura Corporal } \\
\hline Baixo & - & - & - & - \\
\hline $\begin{array}{l}\text { Adequado } \\
\quad \text { Moderadamente acima }\end{array}$ & $8(66,7)$ & $7(63,6)$ & $5(45,4)$ & $20(58,8)$ \\
\hline Excesso & $3(25,0)$ & $1(9,1)$ & $2(18,2)$ & $6(17,6)$ \\
\hline Obesidade & - & $3(27,3)$ & $2(18,2)$ & $5(14,7)$ \\
\hline CMB & $1(8,3)$ & - & $2(18,2)$ & $3(8,8)$ \\
\hline \multicolumn{5}{|l|}{ Desnutrição grave } \\
\hline Desnutrição moderada & - & - & - & - \\
\hline \multicolumn{5}{|l|}{ Desnutrição leve } \\
\hline Eutrofia & - & $1(9,1)$ & - & $1(2,9)$ \\
\hline $\mathrm{AMBC}$ & $4(33,3)$ & $4(36,4)$ & $4(36,4)$ & $12(35,3)$ \\
\hline $\begin{array}{l}\text { Normal } \\
\text { Desnutrição leve/moder }\end{array}$ & $8(66,7)$ & $6(54,5)$ & $7(58,3)$ & $21(61,8)$ \\
\hline \multirow[t]{3}{*}{ Desnutrição grave } & $7(58,3)$ & $5(45,4)$ & $6(54,5)$ & $18(53,0)$ \\
\hline & $3(25,0)$ & $2(18,2)$ & $1(9,1)$ & $6(17,6)$ \\
\hline & $2(16,7)$ & $4(36,4)$ & $4(36,4)$ & $10(29,4)$ \\
\hline
\end{tabular}


Tabela 6 - Perfil lipídico e glicêmico de indivíduos onívoros, ovolactovegetarianos e veganos.

Criciúma - Santa Catarina. 2018

\begin{tabular}{|c|c|c|c|c|}
\hline Variável & $\begin{array}{c}\text { Onívoros } \\
\text { (n=12) } \\
f(\%)\end{array}$ & $\begin{array}{c}\text { Ovolacto } \\
\begin{array}{c}(n=11) \\
f(\%)\end{array}\end{array}$ & $\begin{array}{c}\text { Veganos } \\
(n=11) \\
f(\%)\end{array}$ & $\begin{array}{l}\text { Total } \\
(n=34) \\
f(\%) \\
\end{array}$ \\
\hline \multicolumn{5}{|l|}{ Colesterol Total' } \\
\hline$<190 \mathrm{mg} / \mathrm{dL}$ & $5(41,7)$ & $11(100)$ & $9(81,8)$ & $25(73,5)$ \\
\hline$>190 \mathrm{mg} / \mathrm{dL}$ & $7(58,3)$ & 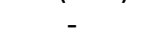 & $2(18,2)$ & $9(26,5)$ \\
\hline \multicolumn{5}{|l|}{$H^{\prime} L^{1}$} \\
\hline$>40 \mathrm{mg} / \mathrm{dL}$ & $12(100)$ & $9(81,8)$ & $9(81,8)$ & $30(88,2)$ \\
\hline$<40 \mathrm{mg} / \mathrm{dL}$ & & $2(18,2)$ & $2(18,2)$ & $4(11,8)$ \\
\hline \multicolumn{5}{|l|}{ Triglicerídeos' } \\
\hline$<150 \mathrm{mg} / \mathrm{dL}$ & $12(100)$ & $11(100)$ & $11(100)$ & $34(100)$ \\
\hline \multicolumn{5}{|l|}{ LDL $^{1}$} \\
\hline$<130 \mathrm{mg} / \mathrm{dL}$ & $9(75,0)$ & $11(100)$ & $10(90,9)$ & $30(88,2)$ \\
\hline$>130 \mathrm{mg} / \mathrm{dL}$ & $3(25,0)$ & - & $1(9,1)$ & $4(11,8)$ \\
\hline \multicolumn{5}{|l|}{ Glicose em Jejum ${ }^{2}$} \\
\hline $\begin{array}{l}<100 \mathrm{mg} / \mathrm{dL} \\
\quad \geq 100 \mathrm{mg} / \mathrm{dL} \mathrm{e}<126 \mathrm{mg} / \mathrm{dL}\end{array}$ & $6(50,0)$ & $11(100)$ & $9(81,8)$ & $26(76,5)$ \\
\hline$\geq 126$ mg/dL & $6(50,0)$ & - & $2(18,2)$ & $8(23,5)$ \\
\hline & & - & & \\
\hline
\end{tabular}

Fonte: Elaborada pelos autores.

HDL: High - Density Lipoprotein. LDL: Low - Density Lipoprotein cholesterol.

1 Diretriz Brasileira de Dislipidemia (2017).

${ }^{2}$ Diretriz da Sociedade Brasileira de Diabetes (2017).

Tabela 7 - Classificação hematológica de indivíduos onívoros, ovolactovegetarianos e veganos. Criciúma - Santa Catarina. 2018

\begin{tabular}{|c|c|c|c|c|}
\hline Variável & $\begin{array}{c}\text { OOnívoros } \\
\begin{array}{c}(n=12) \\
f(\%)\end{array}\end{array}$ & $\begin{array}{c}\text { OOvolacto } \\
(n=11) \\
f(\%)\end{array}$ & $\begin{array}{c}\text { VVeganos } \\
(n=11) \\
f(\%) \\
\end{array}$ & $\begin{array}{c}\text { TTotal } \\
(n=34) \\
f(\%)\end{array}$ \\
\hline \multicolumn{5}{|c|}{ Hemácias (milhões/mm³) } \\
\hline Normal & $10(83,3)$ & $11(100)$ & $10(90,9)$ & $31(91,2)$ \\
\hline Abaixo & $2(16,7)$ & - & $1(9,1)$ & $3(8,8)$ \\
\hline Acima & - & - & - & - \\
\hline \multicolumn{5}{|c|}{ Hemoglobina (g/dL) } \\
\hline Normal & $7(58,33)$ & $8(72,7)$ & $8(72,7)$ & $23(67,6)$ \\
\hline Abaixo & $5(41,66)$ & $3(27,3)$ & $3(27,3)$ & $11(32,3)$ \\
\hline Acima & - & - & - & - \\
\hline
\end{tabular}

Fonte: Elaborada pelos autores.

Tabela 8 - Níveis de vitamina B12 de indivíduos ovolactovegetarianos e veganos (dados de prontuário). Criciúma - Santa Catarina. 2018

\begin{tabular}{lccc}
\hline Variável* $^{*}$ & $\begin{array}{c}\text { Ovolacto } \\
(\mathbf{n = 1 1})\end{array}$ & $\begin{array}{c}\text { Veganos } \\
(\mathbf{n = 1 1 )}\end{array}$ & $\begin{array}{c}\text { Total } \\
(\mathbf{n}=\mathbf{2 2})\end{array}$ \\
\hline Vitamina B12 $(\mathrm{pg} / \mathrm{mL})$ & $340,27 \pm 67,76$ & $385,45 \pm 151,51$ & $362,86 \pm 119,51$ \\
\hline
\end{tabular}

Fonte: Elaborada pelos autores. 
servado desnutrição grave, ou seja, uma quantidade inferior ao normal de massa magra em todos os grupos, prevalecendo mais entre ovolactovegetarianos e veganos, com $36,4 \%$ em cada um (Tabela 5 ).

Em referência aos níveis de colesterol total, verificou-se que a maioria dos ovolactovegetarianos e veganos apresentou colesterol normal, enquanto um maior número de onívoros mostrou colesterol acima do adequado. Já nos níveis de HDL a maioria está dentro da normalidade; somente nos ovolactos e veganos obteve-se níveis abaixo do normal. No que se refere ao LDL e triglicerídeos, a maior parte está dentro da normalidade, não havendo diferenças entre os grupos. Quanto aos resultados da glicemia, foi observado que metade do grupo estava com risco aumentado para diabetes (Tabela 6).

No hemograma pode-se observar nos resultados maior predominância de indivíduos onívoros com hemoglobina abaixo do ideal, tendo como indicativo a anemia. Vale ressaltar que foram considerados como ponto de corte os indicativos estabelecidos pela World Health Organization (2001), sendo a hemoglobina $<12 \mathrm{~g} / \mathrm{dL}$ em mulheres e $<13 \mathrm{~g} / \mathrm{dL}$ em homens.

Em consideração à vitamina B12 analisada nos indivíduos vegetarianos, conforme evidenciado na Tabela 8, é interessante observar que a média se encontra dentro dos valores de referência ideais. Não foi possível analisar os níveis séricos de vitamina B12 no grupo dos onívoros pela falta de realização de exames periódicos sobre esta vitamina nos mesmos; assim, foram apresentados apenas os resultados do público vegetariano.

\section{DISCUSSÃO}

Com o aumento da popularidade das dietas à base de plantas, o vegetarianismo vem ganhando reconhecimento e já é considerado uma escolha dietética saudável, posto que pode fornecer benefícios à saúde e atuar na prevenção e tratamento de certas doenças. A American Dietetic Association (ADA) afirma que dietas vegetarianas ou veganas, bem planejadas, são nutricionalmente adequadas durante todas as fases da vida, incluindo gravidez, lactação, infância, adolescência e para atletas (MELINA et al., 2016).

$A$ dieta vegetariana tem sido associada à maior perda de peso, menor IMC, como também menor CC (HAUSCHILD et al., 2015). Phillips et al. (2004) avaliaram antropometricamente indivíduos que seguiram dieta vegetariana por seis meses e observaram reduções significativas nas dobras cutâneas, incluindo a CC. Chiu et al. (2015) também pesquisaram vegetaria- nos e não vegetarianos, e notaram valores mais baixos de CC nos vegetarianos. Resultado semelhante ocorreu no presente estudo, posto que nos onívoros foi observado risco moderado maior para complicações metabólicas associadas à obesidade.

Em relação ao IMC, Agrawal et al. (2014), ao investigarem 156.317 adultos de 20 a 49 anos com dietas vegetariana e não vegetariana, verificaram que a média do IMC foi menor em veganos $\left(20,5 \mathrm{~kg} / \mathrm{m}^{2}\right)$ e onívoros $\left(20,7 \mathrm{~kg} / \mathrm{m}^{2}\right)$ e maior nas dietas ovolactovegetarianas $\left(21,0 \mathrm{~kg} / \mathrm{m}^{2}\right)$. Já no estudo realizado por Sofi et al. (2018), não foram encontradas diferenças significativas de IMC dos vegetarianos e onívoros pesquisados, e todos apresentaram resultados adequados de peso e IMC. O mesmo aconteceu neste estudo, que mostrou IMC na maioria dos avaliados e sem diferenças entre os grupos.

A avaliação da composição corporal foi importante para diagnosticar com mais precisão o estado nutricional dos pesquisados, por meio da quantificação de massa gorda e massa magra. Kim et al. (2012) investigaram 45 vegetarianos e 30 onívoros e observaram que vegetarianos, a longo prazo, tinham percentual de gordura corporal menor $(21,6 \pm 6,4 \%)$ do que os onívoros $(25,4 \pm 4,6 \%)$. Em outra pesquisa, feita por Slywitch (2010), foi avaliado, comparativamente, o percentual de massa magra e gorda de vegetarianos e não vegetarianos e não encontrou diferenças significativas entres os grupos.

No presente estudo, todavia, verificou-se que gordura corporal acima do ideal nos vegetarianos pode estar relacionada aos motivos de esses indivíduos terem adotado a dieta vegetariana. Ou seja, possivelmente as escolhas alimentares podem não estar nutricionalmente adequadas ou, ainda, os sujeitos apresentem um estilo de vida mais sedentário. Hábitos alimentares e atividade física não foram avaliados, podendo ser um fator limitante dessa pesquisa.

O perfil lipídico, cuja investigação analisou os níveis de Colesterol Total, LDL, HDL e Triglicerídeos, torna-se relevante para identificar possíveis riscos para doenças cardiovasculares, e os hábitos alimentares parecem desempenhar um papel importante no desenvolvimento da aterosclerose. Dessa forma, a dieta vegetariana tem sido associada a menores valores de $\mathrm{CT}$, TG e LDL quando comparada à dieta não vegetariana. De Biase et al. (2007) obtiveram essa confirmação quando investigaram 76 pessoas, divididas entre dieta onívora, ovolacto, lacto e vegana. Em relação ao HDL, todavia, não houve diferenças significativas nas amostras. Achado semelhante ocorreu no estudo de Kim et al. (2012), mas os níveis de TG não diferiram entre os 
grupos. Outro estudo verificou que uma dieta lactovegetariana com baixo teor de gordura, a curto prazo, produz reduções favoráveis no colesterol total, LDL e triglicerídeos, porém também produz uma diminuição no HDL (QUILES et al., 2015).

Nesta pesquisa é relevante observar que $100 \%$ dos ovolactovegetarianos apresentaram níveis normais de CT, LDL e TG, e a maior parte dos veganos também mostrou normalidade nas três dosagens. Os resultados de HDL não tiveram diferenças significativas entre os três grupos, sendo semelhante ao resultado de outros trabalhos (RIBEIRO et al., 2015).

A glicemia em jejum é utilizada para diagnosticar previamente uma glicemia de jejum alterada, pré-diabetes e diabetes. Nesta pesquisa em questão, também se verificou se o tipo de dieta influencia, e foi observado diferenças importantes entre os grupos onívoros e vegetarianos. Agrawal et al. (2014) verificaram resultado semelhante em estudo que buscou uma associação entre o consumo de uma dieta vegetariana e a ocorrência de diabetes tipo 2 em uma amostra de 156.317 participantes, com idade de 20 a 49 anos. Indivíduos que aderiram a qualquer tipo de dieta vegetariana, exceto a vegana, tiveram estatisticamente menores chances de diabetes. Ribeiro et al. (2015), em estudo semelhante, analisaram o perfil bioquímico de indivíduos vegetarianos e onívoros e não encontraram diferenças nos resultados de glicemia entre eles. 0 mesmo ocorreu em pesquisa anterior realizada por Slywitch (2010), na qual os valores apresentados em ambos os grupos estavam dentro da normalidade.

O perfil glicêmico positivo observado nos vegetarianos aqui avaliados, pode estar relacionado pela maioria ter apresentado Circunferência da Cintura menor, o que diminui os riscos para alterações metabólicas, como a hiperglicemia. Esses resultados estão de acordo com estudos anteriores, que associam a dieta vegetariana ao risco reduzido para diabetes, redução na glicemia e na Circunferência da Cintura (YOKOYAMA et al., 2014; TURNER-MCGRIEVY et al., 2014).

O hemograma foi utilizado para verificar alterações na hemoglobina com possível indicativo de anemia e comparando os valores entre vegetarianos e não vegetarianos. Anteriormente, estudos, como de Friedrich (2010), analisou o perfil bioquímico por meio de exames laboratoriais, incluindo o hemograma, de 20 vegetarianos e 29 não vegetarianos e constatou resultados semelhantes de hemoglobina nos dois grupos, com níveis dentro da normalidade. Da mesma forma, Shridhar et al. (2014) avaliaram os níveis séricos de hemoglobina em vegetarianos e onívoros e encontraram os valores de $12,7 \pm 1,8 \mathrm{~g} / \mathrm{dL}$ em vegetarianos e 13,3 $\pm 3,7 \mathrm{~g} / \mathrm{dL}$ em onívoros $(\mathrm{p}<0,0001)$. Hackbarth (2015) observou, entretanto, valores menores de hemoglobina nos vegetarianos em relação aos onívoros, pois foram feitas análises bioquímicas em 40 vegetarianos e 40 onívoros e $14,8 \%$ dos vegetarianos apresentaram hemoglobina reduzida, contra $5,3 \%$ dos onívoros.

Neste estudo notou-se um resultado diferente. Com isso, apesar de outros exames serem necessários para detectar e classificar o tipo de anemia, foi possível perceber que esta pode estar presente tanto nos vegetarianos quanto nos onívoros, não sendo relacionada meramente à ingestão ou não de carnes.

Um dos tipos de anemia - a megaloblástica - é ocasionada pela deficiência de vitamina B12. A consequência clínica desta carência inclui, além da anemia megaloblástica, progressiva doença neurológica do nervo central e sistema periférico, e hiper-homocisteinemia, fator de risco para doença cardiovascular. Por ser comumente assinalada como risco em dietas vegetarianas, decidiu-se avaliar os níveis séricos no grupo dos vegetarianos.

Sabe-se que a vitamina B12 é uma vitamina hidrossolúvel encontrada em quantidades apenas em alimentos de origem animal. Se o consumo destes é muito baixo ou ausente, a presença em alimentos vegetais torna-se essencial, seja por meio de suplementos ou alimentos fortificados. Pesquisas documentaram que a prevalência de deficiência é relativamente alta entre vegetarianos. Veganos que não ingerem suplementos de vitamina B12 foram considerados de alto risco. Independentemente do tipo de dieta vegetariana adotada, é indicado que os vegetarianos devem ser avaliados quanto à deficiência de vitamina B12 (PAWLAK et al., 2013).

Slywitch (2010) avaliou os níveis séricos de vitamina B12 de 29 vegetarianos comparando com 22 onívoros, e constatou que ambos os grupos apresentaram resultados parecidos, com uma média de $387,7 \mathrm{pg} / \mathrm{ml}$ nos onívoros e $350,2 \mathrm{pg} / \mathrm{ml}$ nos vegetarianos. Em uma revisão sistemática de literatura com base na concentração sanguínea de B12 entre vegetarianos, a deficiência foi verificada variando de $0 \%$ para $86,5 \%$ entre adultos e idosos, até $45 \%$ em lactentes, de $0 \%$ para $33,3 \%$ em crianças e adolescentes e de $17 \%$ a $39 \%$ entre mulheres grávidas. Recentemente, outros estudos demonstraram uma diminuição da vitamina B12 entre vegetarianos (PAWLAK et al., 2013; PARK et al., 2015).

Nesta pesquisa, partindo do padrão de normalidade estabelecido pela OMS para vitamina B12 de 480 $\mathrm{pg} / \mathrm{mL}$ e para deficiência os valores menores de 150 $\mathrm{pg} / \mathrm{mL}$, certificou-se que os níveis estão apropriados. 
Não foi questionado aos participantes sobre a existência de sintomas que pudessem estar relacionados à deficiência de B12, ou se fazem uso de suplementos, nem foram realizados exames específicos para detectar anemia por deficiência de outras vitaminas. Apesar disso, diferentemente dos estudos anteriores suprarrelatados, sugere-se que o fato de eles estarem com níveis adequados pode ter relação com o tempo de vegetarianismo recente e ainda apresentarem boas reservas no organismo.

\section{CONCLUSÃO}

Os autores concordam com a literatura, quando se nota que há influência da dieta vegetariana em diversos parâmetros dos seus praticantes. Mediante este trabalho, podemos concluir que os vegetarianos apresentam diferenças sobre o perfil lipídico e glicêmico quando comparados aos onívoros, bem como na composição corporal, uma vez que a circunferência da cintura pareceu diferente entre os grupos, e os vegetarianos apresentaram resultados inferiores ao grupo onívoro.

Como limitações do trabalho, apontamos o público restrito de vegetarianos na cidade da pesquisa (Criciúma, Santa Catarina). Apesar do grande crescimento dos adeptos a uma dieta vegetariana, os números na região ainda são pequenos comparados a outros trabalhos científicos. A relevância deste trabalho, contudo, se dá pela temática inovadora, bem como por relatar o público vegetariano em Santa Catarina. Para próximos trabalhos, sugere-se a possibilidade de realizar o exame de vitamina B12 com a coleta de sangue do momento, posto que o mesmo fora coletado do prontuário por falta de acesso aos materiais para a realização dos exames.

\section{REFERÊNCIAS}

ABEP. Associação Brasileira de Empresas de Pesquisa. Critério de classificação econômica Brasil. 2008. Disponível em: www.abep.org. Acesso em: 14 fev. 2020.

AGRAWAL, Sutapa et al. Type of vegetarian diet, obesity and diabetes in adult Indian population. Nutrition Journal, n. 13, v. 1, p. 89, 2014.

BLACKBURN, George L.; THORNTON, Paul A. Nutritional assessment of the hospitalized patient. The Medical Clinics of North America, v. 63, n. 5, p. 11.103-11.115, 1979.

CHIU, Yi F. et al. Cross-sectional and longitudinal comparisons of metabolic profiles between vegetarian and non-vegetarian subjects: a matched cohort study. British Journal of Nutrition, v. 114, n. 8, p. 1.313-1.320. 2015.
DE BIASE, Simone Grigoletto et al. Dieta vegetariana e níveis de colesterol e triglicérides. Arq Bras Cardiol, v. 88, n. 1, p. 35-39, 2007.

FRIEDRICH, Luana. Avaliação do estado nutricional de ferro em indivíduos vegetarianos e onívoros. 2010. Dissertação (Mestrado) - Universidade Federal de São Paulo, São Paulo, 2010.

HACKBARTH, L. Estado nutricional de vegetarianos e onivoros usuários de restaurantes universitários. Dissertação (Mestrado) - Universidade Federal do Paraná, Curitiba, 2015.

HAUSCHILD, Lucas et al. Estado nutricional e qualidade da dieta em indivíduos vegetarianos estritos e não-estritos. $R e$ vista Uningá Review, v. 23, n. 2, p. 18-24, 2015.

IBOPE. Instituto Brasileiro de Opinião Pública e Estatística. Pesquisa de opinião pública sobre vegetarianismo. 2018. Disponível em: www.ibopeinteligencia.com. Acesso em: 14 fev. 2020.

KIM, M. K. et al. Long-term vegetarians have low oxidative stress, body fat, and cholesterol levels. Nutrition Research and Practic, v. 6, n. 2, p. 155-161, 2012.

MARSH, Kate et al. Health implications of a vegetarian diet: A review. American Journal of Lifestyle Medicine, v. 6, n. 3, p. 250-267, 2012.

MARTINS, Cristina. Avaliação do estado nutricional e diagnóstico. Curitiba: NutroClínica. 2008.

MELINA, Vesanto et al. Position of the Academy of Nutrition and Dietetics: Vegetarian Diets. Journal of the Academy of Nutrition and Dietetics, v. 116, n. 12, p. 1.970-1.980, 2016.

MOTTA, Valter Teixeira. Bioquímica clínica para laboratório: princípios e interpretações. 5. ed. Rio de Janeiro: Medbook, 2009. 383 p.

PARK, Jong-eun et al. Distribution of seven N-nitrosamines in food. Toxicological Research, v. 31, n. 3, p. 279, 2015.

PAWLAK, R. et al. How prevalent is vitamin B12 deficiency among vegetarians? Nutrition Reviews, v. 71, n. 2, p. 101117, 2013.

PHILLIPS, F. et al. Effect of changing to a self-selected vegetarian diet on anthropometric measurements in UK adults. Journal of Human Nutrition and Dietetics, v. 17, n. 3, p. 249255, 2004.

POLLOCK, Michel; WILMORE, Jack. Exercícios na saúde e na doença. 2. ed. Rio de Janeiro: Medsi, 1993.

QUILES, Laura et al. Efectos a corto plazo en el perfil lipídico y la glucemia de una dieta vegetariana baja en grasa. Nutrición Hospitalaria, v. 32, n. 1, p. 156-164, 2015.

RIBEIRO, Marcela Ferreira et al. Ingestão alimentar, perfil bioquímico e estado nutricional entre vegetarianos e não vegetarianos. Arquivos de Ciências da Saúde, 22 (3), p. 5863, 2015.

SAMBOL, S. Z. et al. Haematological, biochemical and bone density parameters in vegetarians and non-vegetarians. West Indian Medical Journal, v. 58, n. 6, p. 512-517, 2009. 
SHRIDHAR, K. et al. Ebrahim S. Nutritional profile of Indian vegetarian diets-the Indian Migration Study (IMS). Nutrition Jornal, v. 13, n. 1, p. 55, 2014.

SLYWITCH, Eric. Avaliação do Estado metabólico e nutricional de indivíduos vegetarianos e onívoros. 2010. Dissertação (Mestrado) - Universidade Federal de São Paulo, São Paulo, 2010.

SOFI, F. et al, Low-Calorie Vegetarian Versus Mediterranean Diets for Reducing Body Weight and Improving Cardiovascular Risk Profile: Cardiveg Study (Cardiovascular Prevention With Vegetarian Diet). Circulation, n. 137, v. 11, p. 1.103-1.113, 2018.

TEIXEIRA, Rita de Cássia Moreira de Almeida et al. Risco cardiovascular em vegetarianos e onívoros: um estudo comparativo. Arquivos Brasileiros de Cardiologia, v. 89, n. 4, p. 237-244, 2007.

TURNER-MCGRIEVY, Grabrielle; HARRIS M. Key elements of plant-based diets associated with reduced risk of metabolic syndrome. Current Diabetes Reports, v. 14, n. 9, p. 524, 2014.

VINAGRE, Juliana Christiano de Matos. Efeitos da dieta vegetariana no metabolismo de quilomícrons e aspectos qualitativos da lipoproteína de alta densidade (HDL). 2010. Tese (Doutorado) - Faculdade de Medicina da Universidade de São Paulo, São Paulo, 2010.

WHO. World Health Organisation. Organização Onis, M.; Onyango, A. W.; Borghi, E.; Garza, C.; Yang, H.; WHO Multicentre Growth Reference Study Group, 1995.

WHO. World Health Organization. Obesity: preventing and managing the global epidemic. Geneva: Program of Nutrition, Family and Reproductive Health, 1998.

WHO. World Health Organization. Iron deficiency anemia: assessment, prevention and control. A guide for programme managers. WHO/NDH/01.3. Geneve: WHO/NHD, 2001.

YOKOYAMA, Y. et al. Vegetarian Diets and Blood Pressure. Jama Internal Medicine, [S.I.], v. 174, n. 4, p. 577-587, 2014. 